\title{
The expression and clinical significance of GADD45A in breast cancer patients
}

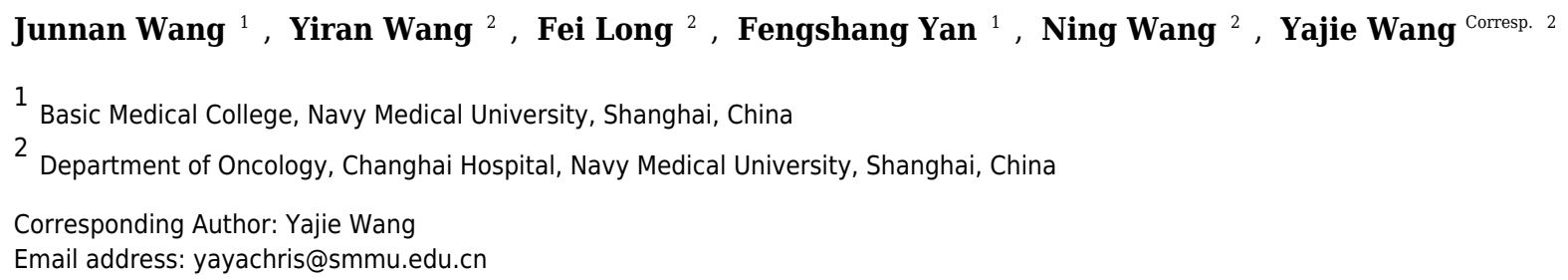

Background. Growth arrest and DNA-damage-inducible protein 45 alpha (GADD45A) was previously found to be associated with risk of several kinds of human tumors. Here, we studied the expression and clinical significance of GADD45A in breast cancer. Methods. We performed an immunohistochemical study of GADD45A protein from 419 breast cancer tissues and 116 adjacent non-neoplastic tissues. Results. Significantly high GADD45A expression were observed in breast cancer tissues compared with adjacent non-neoplastic tissues $(P<0.001)$ and were independently correlative with estrogen receptor negative $(P=0.028)$ and high Ki-67 index $(P<0.001)$. Kaplan-Meier survival analysis revealed that patients with high GADD45A expression levels had a worse long-term prognosis in triple negative breast cancer $(P=0.041)$, but it was not an independent prognostic factor in multivariate analysis $(P=0.058)$. Conclusions. GADD45A expression levels are significantly correlative with estrogen receptor status and Ki-67 index in human breast cancer. Patients with triple negative breast cancer might be stratified into high risk and low risk groups based on the GADD45A expression levels. 
1 The expression and clinical significance of GADD45A in breast cancer patients

\section{Junnan Wang ${ }^{1+}$, Yiran Wang ${ }^{2+}$, Fei Long ${ }^{2}$, Fengshang Yan ${ }^{1}$, Ning Wang ${ }^{2}$, Yajie Wang}

3 Corresp. 2

$4 \quad 1$ Basic Medical College, Naval Medical University, 200433 Shanghai, China

52 Department of Oncology, Changhai Hospital, Naval Medical University, 200433 Shanghai,

6 China;

7 Junnan Wang and Yiran Wang contributed equally to this work.

8 Corresponding Author: Yajie Wang

$9 \quad$ Email address: wangyjscholar@sina.com

10

11

12

13

14

15

16

17

18 


\section{Abstract:}

22 Background. Growth arrest and DNA-damage-inducible protein 45 alpha (GADD45A) was

23 previously found to be associated with risk of several kinds of human tumors. Here, we studied 24 the expression and clinical significance of GADD45A in breast cancer. Methods. We performed

25 an immunohistochemical study of GADD45A protein from 419 breast cancer tissues and 116 26 adjacent non-neoplastic tissues. Results. Significantly high GADD45A expression were observed 27 in breast cancer tissues compared with adjacent non-neoplastic tissues $(P<0.001)$ and were 28 independently correlative with estrogen receptor negative $(P=0.028)$ and high Ki-67 index $29(P<0.001)$. Kaplan-Meier survival analysis revealed that patients with high GADD45A expression 30 levels had a worse long-term prognosis in triple negative breast cancer $(P=0.041)$, but it was not 31 an independent prognostic factor in multivariate analysis $(P=0.058)$. Conclusions. GADD45A 32 expression levels are significantly correlative with estrogen receptor status and Ki-67 index in

33 human breast cancer. Patients with triple negative breast cancer might be stratified into high risk 34 and low risk groups based on the GADD45A expression levels. 


\section{Background}

Breast cancer has become the most common malignant tumor threatening women's health whether in the developed countries or in developing countries. In the United States, there are an estimated 252,710 female cases diagnosed with breast cancer in 2017(Siegel et al. 2017). Meanwhile the incidence of breast cancer in China presents the trend of rising rapidly, over 3.9\% annual percentage change from 2000 to 2011 (Chen et al. 2016). However, the pathogenesis of breast cancer, possibly related to disorder of cell cycle and genetic abnormalities, is not clear yet. Therefore, understanding the connection of biological characteristics and clinicopathological significance is an important aspect for investigating the pathogenesis and identifying therapeutic 51 targets.

Growth arrest and DNA-damage-inducible protein 45 alpha (GADD45A) is a downstream target gene of p53 and BRCA1 (breast cancer susceptibility gene 1), which are two of the most

54 important genes maintaining the stability of the genome and suppressing the development of tumor 55 through various mechanisms(Li et al. 2017b). As a member of GADD45 family of genes that are 56 known as stress sensors, GADD45A modulates the cellular response to a variety of stress 57 conditions, including genotoxic and oncogenic stress(Cretu et al. 2009). And some research 58 showed GADD45A can promote or suppress breast carcinoma development depending on 59 different signaling pathways(Carrier et al. 1999; Miki et al. 1994). 
Additionally, the correlation of GADD45A expression and clinicopathologic factors is not

61 clear in breast cancer thus far. The aim of the current study is to investigate the significance of

62 GADD45A in breast cancer, including the correlation with clinicopathologic factors and long-term

63 survival situation.

2. Materials and Methods

65

66

67

68

69

70

71

72

73

74

\subsection{Tissue samples and clinical data}

The study retrospectively collected 419 cases of tissue specimens from breast cancer patients, undergoing the operation from February 2005 to October 2007, at Changhai Hospital, Shanghai, China. Patients with other primary tumor sites were excluded. Clinicopathologic data was recorded from the patients' medical records including age at diagnosis, molecular classification (ST Gallen), TNM stage (AJCC), pathology types, histological grade (Elston-Ellis grade) and clinical outcomes. Cutoff for positivity was set at $\geq 1 \%$ of cells staining positively for ER or PR. HER-2 status was assessed using the HercepTest ${ }^{\mathrm{TM}}$ according to the manufacturer's instructions. HER-2 positivity was defined as a $3+$ score on IHC in $>10 \%$ of invasive tumor cells. Equivocal IHC cases $(2+$ score or $3+$ score in $\leq 10 \%$ of invasive tumor cells $)$ were submitted to FISH analysis. A ratio of HER-2 signals to chromosome 17 signals $>2.0$ was used as the cutoff to define HER-2 amplification. Ki-67 index greater than $20 \%$ was classified as high. In short, Luminal A was defined as ER+ or PR+, HER2- and low Ki67, Luminal B as ER+ or PR+ and HER2+ or high Ki67; HER2 positive as HER2+ and ER- and PR-; basal-like as HER2-/ER-/PR-. In addition, 116 cases of adjacent non-neoplastic tissue samples were also collected from mammoplasties as a control group. 
81 GADD45A color reaction/positive staining were observed in 419 tumor tissues and 116

82 adjacent non-neoplastic tissues. The study was approved by the Ethics Committee of Biomedicine,

83 Navy Medical University. The samples were used after obtaining informed written consents from

84 all included patients.

\section{$85 \quad 2.2$ Tissue microarray and immunohistochemistry}

The breast cancer tissue microarray (TMA) was obtained from the surgically resected tissues.

Biopsies were fixed in 10\% neutral formalin. TMA blocks were made by tissue arraying instrument

88 (Beecher Instruments, Sun Prairie, WI, USA). Cylinders cores (1.5 $\mathrm{mm}$ in diameter) of tissue

89 blocks were extracted from the center of the tumor, and then arranged into blank recipient paraffin

90 blocks. The TMA blocks were cut into 4- $\mu \mathrm{m}$ sections and treated for immunohistochemistry (IHC).

91 Following deparaffinization and rehydration of the tissues sections, antigen retrieval was

92 performed in the pressure cooker in citrate buffer, $\mathrm{pH} 6.0$, for 30 minutes at a sub-boiling

93 temperature. Endogenous peroxidase was blocked with 3\% peroxide for 10 min. Primary

94 GADD45A antibody (1:200; catno. Sc-792; Santa Cruz Biotechnology, Dallas, TX, USA) were

95 used on tissue sections for immunohistochemical staining. For a negative control, we replaced

96 the primary antibody rabbit immunoglobulin G or normal murine with the same dilution.

97 The GADD45A staining results, independently reviewed and scored in triplicate by two

98 pathologists, were assessed by the staining intensity and the percentage of positively stained cells

99 of each tissue sample. The final GADD45A expression score can be calculated through 
100 multiplying the positive cells percentage score $(0,<10 \% ; 1,10-25 \% ; 2,26-50 \% ; 3,51-75 \%$; and

$1014,>75 \%)$ by the staining intensity score ( 0 , negative; 1 , weak; 2 , moderate; 3 , strong staining). The

102 expression situation of tissue sections were evaluated by the total score ( $>$ five, high expression;

$103 \leq 5$, low expression). To calculate a mean score, total 10 independent fields with high magnification

$104(\times 200)$ were observed.

\section{$105 \quad 2.3$ Statistical analyses}

106 Statistical analyses were conducted using the IBM SPSS Statistics (for Windows, version

107 21.0). The Pearson $\chi 2$ test or Fisher's exact test was used to compare the GADD45A expressions

108 between breast cancer tissues and adjacent non-neoplastic tissues and to confirm the correlations

109 between clinicopathological factors. In the combined analyses, all the datasets were pooled and

110 the odds ratios and P-values were estimated with logistic regression model. To assess the effect of

111 GADD45A expression and other factors on overall survival (OS), different Kaplan-Meier curves

112 were plotted with log-rank test. A multivariate Cox regression model was fitted to test the

113 independent contribution of each variable to the patient's OS. For all data, differences were

114 considered statistically significant when $\mathrm{P}<0.05$. Separate analysis was performed for the subtypes

115 of the breast cancer.

\section{3. Results}

117 3.1. Analysis of clinical data 
118 The color reaction/positive staining of GADD45A was observed in 419 tumor tissue samples

119 and 116 adjacent non-neoplastic tissue samples (figure 1). Among these 419 tumor tissue samples,

$120365(87.1 \%)$ were invasive ductal carcinoma (IDC) and 54 (12.9\%) were non-IDC. As for

121 molecular types, there were $74(17.7 \%)$ for luminal A, $206(49.2 \%)$ for luminal B, $70(16.7 \%)$ for

122 Her-2 and 69 (16.5\%) for triple-negtive (TN) subtype breast cancer. As for histological grades,

123 there were $291(69.5 \%)$ for grade I-II and $128(30.5 \%)$ for III. In addition, there were $113(27.0 \%)$

124 for TNM I, 233 (55.6\%) for TNM II, and 73 (17.4\%) for TNM III, respectively.

$125 \quad$ Figure 1:

126 GADD45A expression levels are elevated in human breast cancer samples compared

127 to adjacent non-neoplastic tissues. Representative images from

128 immunohistochemical analysis of GADD45A on (a) high expression in breast cancer

129 tissues, (b) low expression in breast cancer tissues, (c) high expression in adjacent

130 non-neoplastic tissues and (d) low expression in adjacent non-neoplastic tissues.

131

132

3.2. Expression of GADD45A in breast carcinoma and normal tissues

The cytoplasmic staining for GADD45A was identified in the breast carcinoma tissues and

134 adjacent non-neoplastic tissues. Overall, high expression of GADD45A was found in $72.3 \%$

135 (303/419) cancer tissues and 37.9\% (44/116) adjacent noncancerous tissues. The percentage of

136 high GADD45A expression of breast carcinoma tissues was obviously higher than that of adjacent

137 noncancerous tissues and the difference was statistically significant $(P<0.001$, table 1$)$.

138 3.3. Correlation between GADD45A expression and clinical pathological characteristics 
139 The clinical pathological factors used in the current study include the following: age at

140 diagnosis, TNM stage, pathology types, histological grade, molecular subtypes, ER (estrogen

141 receptor), PR (progesterone receptor), HER2 (human epidermal growth factor receptor-2) and Ki-

14267 index. We found a significant correlation between GADD45A expression and ER status, Ki-67

143 index, histological grade and molecular subtypes but not between GADD45A expression and age

144 at TNM stage, pathology types, PR and HER2. The high expression rate of GADD45A was 78.0\%

$145(131 / 168)$ in ER negative group which is 68.5\% (172/251) in ER positive group and the difference

146 was statistically significant $(P=0.034$, table 2$)$. The high GADD45A expression rate of Ki-67 index

147 high-level group $(\geq 20 \%)(83.1 \%, 207 / 249)$ was apparently higher than that of Ki-67 index low-

148 level group $(<20 \%)(56.2 \%, 96 / 170),(P<0.001)$. Meanwhile, a positive correlation was found

149 between GADD45A expression and histological grade $(P=0.013)$. It was also found between

150 GADD45A expression and molecular subtypes $(P<0.001)$.

151 ER status, Ki-67 index and histological grade were selected into logistic regression model

152 analysis (Molecular subtype was not included because its effect was similar with ER status). The

153 results from multivariate analysis confirmed that ER negative and Ki-67 $\geq 20 \%$ were closely related

154 to high GADD45A expression (table 3).

155 3.4. Survival Analysis

156 Kaplan-Meier curves and log-rank tests were used to perform survival analysis to identify the

157 correlation between survival and GADD45A expression. The last follow-up was carried out in July

158 2017. Table 4 showed GADD45A expression was not related to survival when all types as total

159 ( $P=0.803)$. However, in survival analysis for TN subtype patients, low GADD45A expression

160 group had a mean survival period of 119.61 months (SE: 10.81, 95\% CI: 98.43 140.79). Howeve 
$161 \mathrm{r}$, high GADD45A expression group had a mean survival period of 88.73 months (SE: $7.78,95 \%$

162 CI: $73.48 \sim 103.97)(P=0.041)$. Moreover, the 5-year and 10-year survival rate were $94.7 \%$ and 8

$1630.5 \%$ for high GADD45A expression group while $79.7 \%$ and $68.5 \%$ for low GADD45A

164 expression group respectively. Kaplan-Meier survival curves were performed in figure 2.

165 In the univariate analysis, TNM stage and GADD45A were found to be significant 166 independent predictors of OS, respectively (table 5). However, in the multivariate analysis, only

167 TNM stage was identified the significant independent predictor of OS in the TN subtype patients 168 (table 5).

$169 \quad$ Figure 2:

Kaplan-Meier curve of overall survival in relation to GADD45A protein expression in (a) breast cancer; (b) TN subtype breast cancer.

\section{Discussion}

As GADD45A played a role in genomic stability and tumorigenesis, some studies explored

174 to assess the role of GADD45A in various cancers, such as prostate cancer(Reis et al. 2015),

175 ovarian cancer(Yuan et al. 2015), esophageal cancer(Ishiguro et al. 2016) and malignant

176 gliomas(Cui et al. 2017), which revealed different GADD45A expressions in various carcinoma

177 tissues. In a research for esophageal squamous cell carcinoma(ESCC)(Wang et al. 2012) and

178 another research for breast cancer(Tront et al. 2013), GADD45A protein levels were increased

179 significantly in tumor tissues than that in normal tissues. In addition, a recent study showed the

180 expression of GADD45A was upregulated in human non-small-cell lung cancer (NSCLC) cells

181 through a microRNA-138 related post-transcriptional pathway(Li et al. 2017a). 
182 Compared with the previous study for GADD45A in breast cancer(Tront et al. 2013), our 183 study had a larger-sample analysis for the correlations between clinicopathological factors and 184 GADD45A expression. We also showed the correlation with PR and ER respectively while Tront's 185 study only analyzed tissues of $\mathrm{ER}+/ \mathrm{PR}+$. Moreover, our study was the first study estimating the 186 prognostic significance of GADD45A in triple negative breast cancer. We found that GADD45A 187 expression was upregulated in breast cancer tissues. DNA damage was a major source of 188 potentially mutagenic lesions leading carcinogenesis(Gao et al. 2016). GADD45A was shown to 189 participate actively in DNA repair mechanisms(Wingert \& Rieger 2016). Therefore, its 190 upregulation may be due to extensive DNA repair in breast cancer tissues. Beyond that, a study 191 showed that GADD45A promotes Myc-driven breast carcinogenesis by negatively regulating 192 matrix metalloproteinase 10 (MMP10) through GSK-3 $\beta$ (Glycogen synthase kinase 3 beta)/ $\beta$ 193 catenin signaling, resulting in increased tumor vascularization and growth(Tront et al. 2010). In 194 our opinions, this mechanism may result for the high GADD45A expression in breast cancer 195 tissues.

196 In our study, ER negative and high Ki-67 index were demonstrated the significant association 197 with GADD45A expression. ER-negative breast cancer, which had less treatment options such 198 Estrogen-blocking endocrine therapy, had more malignancy behaviors than ER-positive tumors. 199 Ki-67 index was a cellular marker strictly associated with cell proliferation and emerged as a key 200 discriminative feature of malignant phenotype in breast cancer(Pathmanathan et al. 2014). The 201 above factors all suggested high malignant degree. For this, we thought GADD45A may be a 202 biomarker for the malignancy grade of breast cancer tissue. Our results were opposite to the ones 203 presented by Tront et al(Tront et al. 2013) which showed that high GADD45A levels were 204 associated with luminal A and luminal B subtypes but not in triple negative tissues. The 
205 discrepancy could be generated by the higher number of tissues analyzed in our study compared 206 to the previous one (419 vs 45). In addition, we explored the correlation with ER and PR status 207 separately while Tront et al analyzed only tissue where they were both positive, as mentioned 208 above. As a result of these advantages, our study was a more credible and detailed exploration for 209 the correlations between clinicopathological factors and GADD45A expression compared 210 previous study(Tront et al. 2013).

211 For further evaluation, we assessed the effect of GADD45A expression on OS. We 212 demonstrated that patients with higher GADD45A expression levels had a poor long-term 213 prognosis for TN subtype based on Kaplan-Meier survival curves. However, GADD45A 214 expression was not an independent prognosis factor in TN type breast cancer in multivariate 215 analysis. The finding may suggest that GADD45A expression affect the prognosis through the 216 effects of other factors but not the independent action, such as the effect of Ki-67 index, which 217 was reported as an independent variable associated with prognosis in TN subtype patients(Adamo 218 et al. 2017; Pan et al. 2017; Ricciardi et al. 2015). In addition, we found that TNM stage 219 independently influenced the prognosis of TN breast cancer patients. The similar conclusion was 220 determined in previous studies(Han et al. 2017; Yu et al. 2013; Yu et al. 2014).

221 The limitations of current study included the deficiency of follow-up in chemotherapy 222 regimens of patients. It was difficult to analyze the influence of chemotherapy regimen on 223 prognosis of breast cancer. The relationship between different chemotherapy treatments and 224 prognosis in breast cancer should be the focus of the future study and further analyses in biological 225 mechanisms about tumor progression are need. Moreover, the possible selective bias due to the 226 small sample size and inclusion of only postoperative patients may exist in TN breast cancer group. 
227 Despite the limitations, the correlation between GADD45A expression and certain 228 clinicopathological factors in breast cancer can be verified.

\section{5. Conclusions}

230 In present study, we explored the expression and clinical significance of GADD45A in breast

231 carcinoma. High GADD45A was observed in breast cancer tissues compared with adjacent non232 neoplastic tissues. ER negative and high Ki-67 index were correlative with high GADD45A 233 expression. Patients with higher GADD45A expression levels had a poorer long-term prognosis in

234 TN type breast cancer. However, only TNM stage was the independent prognosis factor in TN 235 type breast cancer. Our study suggested that GADD45A may play a role in breast cancer 236 pathogenesis and may eventually help to understand the biological mechanisms affecting tumor 237 progression.

\section{List of abbreviations}

239 DNA-damage-inducible protein 45 alpha (GADD45A)

240 Breast cancer susceptibility gene 1 (BRCA1)

241 Tissue microarray (TMA)

242 Overall survival (OS)

243 Invasive ductal carcinoma (IDC)

244 Triple-negtive (TN)

245 Estrogen receptor (ER)

246 Progesterone receptor (PR)

247 Human epidermal growth factor receptor-2 (HER2) 
248 Esophageal squamous cell carcinoma (ESCC)

249 Non-small-cell lung cancer (NSCLC)

250 Matrix metalloproteinase 10(MMP10)

251 Glycogen synthase kinase 3 beta (GSK-3 3 )

Adamo B, Rita Ricciardi GR, Ieni A, Franchina T, Fazzari C, Sano MV, Angelico G, Michele C, Tuccari G, and Adamo V. 2017. The prognostic significance of combined androgen receptor, E-Cadherin, Ki67 and CK5/6 expression in patients with triple negative breast cancer. Oncotarget 8:76974-76986.

Carrier F, Georgel P, Pourquier P, Blake M, Kontny H, Antinore M, Gariboldi M, Myers T, Weinstein J, Pommier Y, and Fornace A. 1999. Gadd45, a p53-responsive stress protein, modifies DNA accessibility on damaged chromatin. Mol Cell Biol 19:1673-1685.

264 Chen W, Zheng R, Baade P, Zhang S, Zeng H, Bray F, Jemal A, Yu X, and He J. 2016. Cancer statistics in China, 2015. CA Cancer J Clin 66:115-132.

266 Cretu A, Sha X, Tront J, Hoffman B, and Liebermann D. 2009. Stress sensor Gadd45 genes as therapeutic 267 targets in cancer. Cancer Ther 7:268-276. 
268 Cui D, Sajan P, Shi J, Shen Y, Wang K, Deng X, Zhou L, Hu P, and Gao L. 2017. MiR-148a increases glioma

269 cell migration and invasion by downregulating GADD45A in human gliomas with IDH1 R132H

$270 \quad$ mutations. Oncotarget 8:25345-25361.

271 Gao D, Herman JG, and Guo M. 2016. The clinical value of aberrant epigenetic changes of DNA damage 272 repair genes in human cancer. Oncotarget 7:37331-37346.

273 Han ZQ, Gong XM, Zhu B, Wu SW, Yu L, Song WQ, and Wang D. 2017. Expression of ALDH1, MACC1, 274 and KAI1 in the triple-negative breast cancer and their clinical significance. International Journal Of $275 \quad$ Clinical And Experimental Pathology 10:5655-5664.

276

277

278

279

280

281

282

283

284

285

286

287

288

289

290

291

292

293

294

295

296

297

Ishiguro H, Kimura M, Takahashi H, Tanaka T, Mizoguchi K, and Takeyama H. 2016. GADD45A expression is correlated with patient prognosis in esophageal cancer. Oncol Lett 11:277-282.

Li J, Dong J, Li S, Xia W, Su X, Qin X, Chen Y, Ding H, Li H, Huang A, Bai C, Hu T, Wang C, Chu B, and Shao N. 2017a. An alternative microRNA-mediated post-transcriptional regulation of GADD45A by p53 in human non-small-cell lung cancer cells. Sci Rep 7:7153.

Li X-J, Li Z-F, Wang J-J, Han Z, Liu Z, and Liu B-G. 2017b. Effects of microRNA-374 on proliferation, migration, invasion, and apoptosis of human SCC cells by targeting Gadd45a through P53 signaling pathway. Bioscience reports 37.

Miki Y, Swensen J, Shattuck-Eidens D, Futreal P, Harshman K, Tavtigian S, Liu Q, Cochran C, Bennett L, and Ding W. 1994. A strong candidate for the breast and ovarian cancer susceptibility gene BRCA1. Science 266:66-71.

Pan Y, Yuan Y, Liu G, and Wei Y. 2017. P53 and Ki-67 as prognostic markers in triple-negative breast cancer patients. PLoS ONE 12:e0172324.

Pathmanathan N, Balleine RL, Jayasinghe UW, Bilinski KL, Provan PJ, Byth K, Bilous AM, Salisbury EL, and Boyages J. 2014. The prognostic value of Ki67 in systemically untreated patients with nodenegative breast cancer. J Clin Pathol 67:222-228.

Reis I, Ramachandran K, Speer C, Gordian E, and Singal R. 2015. Serum GADD45a methylation is a useful biomarker to distinguish benign vs malignant prostate disease. Br J Cancer 113:460-468.

Ricciardi GR, Adamo B, Ieni A, Licata L, Cardia R, Ferraro G, Franchina T, Tuccari G, and Adamo V. 2015. Androgen Receptor (AR), E-Cadherin, and Ki-67 as Emerging Targets and Novel Prognostic Markers in Triple-Negative Breast Cancer (TNBC) Patients. PLoS ONE 10:e0128368.

Siegel R, Miller K, and Jemal A. 2017. Cancer Statistics, 2017. CA Cancer J Clin 67:7-30.

PeerJ reviewing PDF | (2018:02:23923:1:1:NEW 8 Jun 2018) 
298 Tront J, Huang Y, Fornace A, Fornace A, Hoffman B, and Liebermann D. 2010. Gadd45a functions as a 299 promoter or suppressor of breast cancer dependent on the oncogenic stress. Cancer Res 70:9671$300 \quad 9681$.

301 Tront J, Willis A, Huang Y, Hoffman B, and Liebermann D. 2013. Gadd45a levels in human breast cancer 302 are hormone receptor dependent. J Transl Med 11:131.

303

304

305

306

307

308 309

310 311

312

313

314

315

316

317

318

Wang B, Yin B, He B, Chen C, Zhao M, Zhang W, Xia Z, Pan Y, Tang J, Zhou X, and Yin N. 2012. Overexpression of DNA damage-induced $45 \alpha$ gene contributes to esophageal squamous cell cancer by promoter hypomethylation. J Exp Clin Cancer Res 31:11.

Wingert S, and Rieger MA. 2016. Terminal differentiation induction as DNA damage response in hematopoietic stem cells by GADD45A. Exp Hematol 44:561-566.

Yu GZ, Liu GH, Dong J, and Jin YY. 2013. Clinical implications of CIP2A protein expression in breast cancer. Medical Oncology 30.

Yu H, Li H, Qian H, Jiao X, Zhu XW, Jiang XQ, Dai GH, and Huang JX. 2014. Upregulation of miR-301a correlates with poor prognosis in triple-negative breast cancer. Medical Oncology 31.

Yuan C, Liu X, Liu X, Yang N, Liu Z, Yan S, Shen K, and Kong B. 2015. The GADD45A (1506T>C) Polymorphism Is Associated with Ovarian Cancer Susceptibility and Prognosis. PLoS ONE 10:e0138692.

\section{Figure legends}

\section{Figure 1:}

GADD45A expression levels are elevated in human breast cancer samples compared to adjacent non-neoplastic tissues. Representative images from immunohistochemical analysis of GADD45A on (a) high expression in breast cancer tissues, (b) low expression in breast cancer tissues, (c) high expression in adjacent non-neoplastic tissues and (d) low expression in adjacent non-neoplastic tissues. 
$324 \quad$ Figure 2:

325 Kaplan-Meier curves of overall survival in relation to GADD45A protein expression in

$326 \quad$ (a) breast cancer; (b) TN subtype breast cancer. 
Table $\mathbf{1}$ (on next page)

Expression of GADD45A breast carcinoma tissues and adjacent non-neoplastic tissues. 


\section{Table 1:}

2 Expression of GADD45A breast carcinoma tissues and

3 adjacent non-neoplastic tissues.

\begin{tabular}{|c|c|c|c|c|c|}
\hline \multirow{2}{*}{ Characteristics } & \multicolumn{2}{|c|}{ GADD45A Expression } & \multirow{2}{*}{ Total } & \multirow{2}{*}{$\chi^{2}$} & \multirow{2}{*}{$P$} \\
\hline & High (\%) & Low $(\%)$ & & & \\
\hline $\begin{array}{c}\text { breast carcinoma } \\
\text { tissues }\end{array}$ & $303(72.3)$ & $116(27.7)$ & 419 & & \\
\hline $\begin{array}{c}\text { adjacent non-neoplastic } \\
\text { tissues }\end{array}$ & $44(37.9)$ & $72(62.1)$ & 116 & 47.125 & $<0.001$ \\
\hline
\end{tabular}

4

5 


\section{Table 2 (on next page)}

Correlation between high/low GADD45A expression and clinic pathological factors in 419 breast cancer tissues.

IDC, invasive ductal carcinoma; ER, estrogen receptor; PR, progesterone receptor; HER2, human epidermal growth factor receptor 2 . 
$1 \quad$ Table 2 (Red parts for corrections or additions):

2 Correlation between high/low GADD45A expression and clinic pathological factors in 3419 breast cancer tissues.

\begin{tabular}{|c|c|c|c|c|c|}
\hline \multirow{2}{*}{ Characteristics } & \multicolumn{2}{|c|}{ GADD45A Expression } & \multirow{2}{*}{ Total } & \multirow{2}{*}{$\chi^{2}$} & \multirow{2}{*}{$P$} \\
\hline & High (\%) & Low (\%) & & & \\
\hline \multicolumn{6}{|l|}{ Age } \\
\hline \multicolumn{6}{|l|}{ at diagnosis(years) } \\
\hline$\leq 50$ & $119(70.0)$ & $51(30.0)$ & 170 & & \\
\hline$>50$ & $184(73.9)$ & $65(26.1)$ & 249 & 0.766 & 0.382 \\
\hline \multicolumn{6}{|l|}{ TNM stage } \\
\hline $\mathrm{I}$ & $77(68.1)$ & $36(31.9)$ & 113 & & \\
\hline II & $168(72.1)$ & $65(27.9)$ & 233 & & \\
\hline III & $58(79.5)$ & $15(20.5)$ & 73 & 2.846 & 0.241 \\
\hline \multicolumn{6}{|l|}{ Pathology type } \\
\hline IDC & $269(73.7)$ & $96(26.3)$ & 365 & & \\
\hline non-IDC & $34(63.0)$ & $20(37.0)$ & 54 & 2.708 & 0.100 \\
\hline \multicolumn{6}{|l|}{ Histological grade } \\
\hline I-II & $200(68.7)$ & $91(31.3)$ & 291 & & \\
\hline III & $103(80.5)$ & $25(19.5)$ & 128 & 6.120 & 0.013 \\
\hline
\end{tabular}




\section{Molecular subtype}

\begin{tabular}{|c|c|c|c|c|c|}
\hline Luminal A & $40(54.1)$ & $34(45.9)$ & 74 & & \\
\hline Luminal B & $157(76.2)$ & $49(23.8)$ & 206 & & \\
\hline HER2-enriched & $56(80.0)$ & $14(20.0)$ & 70 & & \\
\hline Triple negative & $50(72.5)$ & $19(27.5)$ & 69 & 23.584 & $<0.001$ \\
\hline \multicolumn{6}{|l|}{ ER } \\
\hline positive & $172(68.5)$ & $79(31.5)$ & 251 & & \\
\hline negative & $131(78.0)$ & $37(22.0)$ & 168 & 4.489 & 0.034 \\
\hline \multicolumn{6}{|l|}{ PR } \\
\hline positive & $163(70.3)$ & $69(29.7)$ & 232 & & \\
\hline negative & $140(74.9)$ & $47(25.1)$ & 187 & 1.098 & 0.295 \\
\hline \multicolumn{6}{|l|}{ HER2 } \\
\hline positive & $134(75.7)$ & $43(24.3)$ & 177 & & \\
\hline negative & $169(69.8)$ & $73(30.2)$ & 242 & 1.760 & 0.185 \\
\hline \multicolumn{6}{|l|}{ Ki-67 } \\
\hline$\geq 20 \%$ & $207(83.1)$ & $42(16.7)$ & 249 & & \\
\hline$<20 \%$ & $96(56.2)$ & $74(43.5)$ & 170 & 35.871 & $<0.001$ \\
\hline
\end{tabular}

4 IDC, invasive ductal carcinoma; ER, estrogen receptor; PR, progesterone receptor;

5 HER2, human epidermal growth factor receptor 2. 


\section{Table 3 (on next page)}

Multivariate analyses by logistic regression analysis. 


\section{Table 3:}

2 Multivariate analyses by logistic regression analysis.

\begin{tabular}{cccccc}
\hline Dependent variable & Independent variable & SE & $P$ value & OR & $95 \%$ CI \\
\hline \multirow{2}{*}{ GADD45A Expression } & ER negative & 0.241 & 0.028 & 1.697 & $1.058-2.723$ \\
& Ki-67 $\geq 20 \%$ & 0.231 & $<0.001$ & 3.861 & $2.455-6.074$ \\
\hline
\end{tabular}

3

4 


\section{Table 4 (on next page)}

Univariate analyses of OS and GADD45A expression of different types patients. 
1

2

3

Table 4:

Univariate analyses of OS and GADD45A expression

of different types patients.

\begin{tabular}{ccc}
\hline Analyses for GADD45A & $\chi 2$ & $\boldsymbol{P}$ Value \\
\hline All types as total & 0.062 & 0.803 \\
Luminal A & 2.011 & 0.156 \\
Luminal B & 3.536 & 0.060 \\
HER2 & 1.565 & 0.211 \\
TN & 4.157 & 0.041 \\
\hline
\end{tabular}

4 


\section{Table 5 (on next page)}

Univariate analyses and multivariate analyses overall survival for TN breast cancer patients. 
1 Table 5:

2 Univariate analyses and multivariate analyses overall survival for $\mathrm{TN}$ breast cancer patients.

\begin{tabular}{|c|c|c|c|c|}
\hline \multirow{2}{*}{ Characteristics } & \multirow{2}{*}{$\begin{array}{c}\text { Univariate analyses } \\
P \text { value }\end{array}$} & \multicolumn{3}{|c|}{ Multivariate analyses } \\
\hline & & HR & $95 \% \mathrm{CI}$ & $P$ value \\
\hline Age $(>50$ vs. $\leq 50)$ & 0.158 & NA & NA & NA \\
\hline TNM stage (III vs I/II) & 0.001 & 3.720 & $1.637-8.452$ & 0.002 \\
\hline Pathology type (non-IDC vs IDC) & 0.445 & NA & NA & $\mathrm{N}$ \\
\hline Histological grade (III vs I/II) & 0.251 & NA & NA & NA \\
\hline Ki-67 (>20\% vs. $\leq 20 \%)$ & 0.060 & NA & NA & NA \\
\hline GADD45A (Positive vs. Negative) & 0.041 & 2.792 & $0.967-8.060$ & 0.058 \\
\hline
\end{tabular}

3 
Figure 1

GADD45A expression levels are elevated in human breast cancer samples compared to adjacent non-neoplastic tissues.

Representative images from immunohistochemical analysis of GADD45A on (A) high expression in breast cancer tissues, (B) low expression in breast cancer tissues, (C) high expression in adjacent non-neoplastic tissues and (D) low expression in adjacent non-neoplastic tissues.

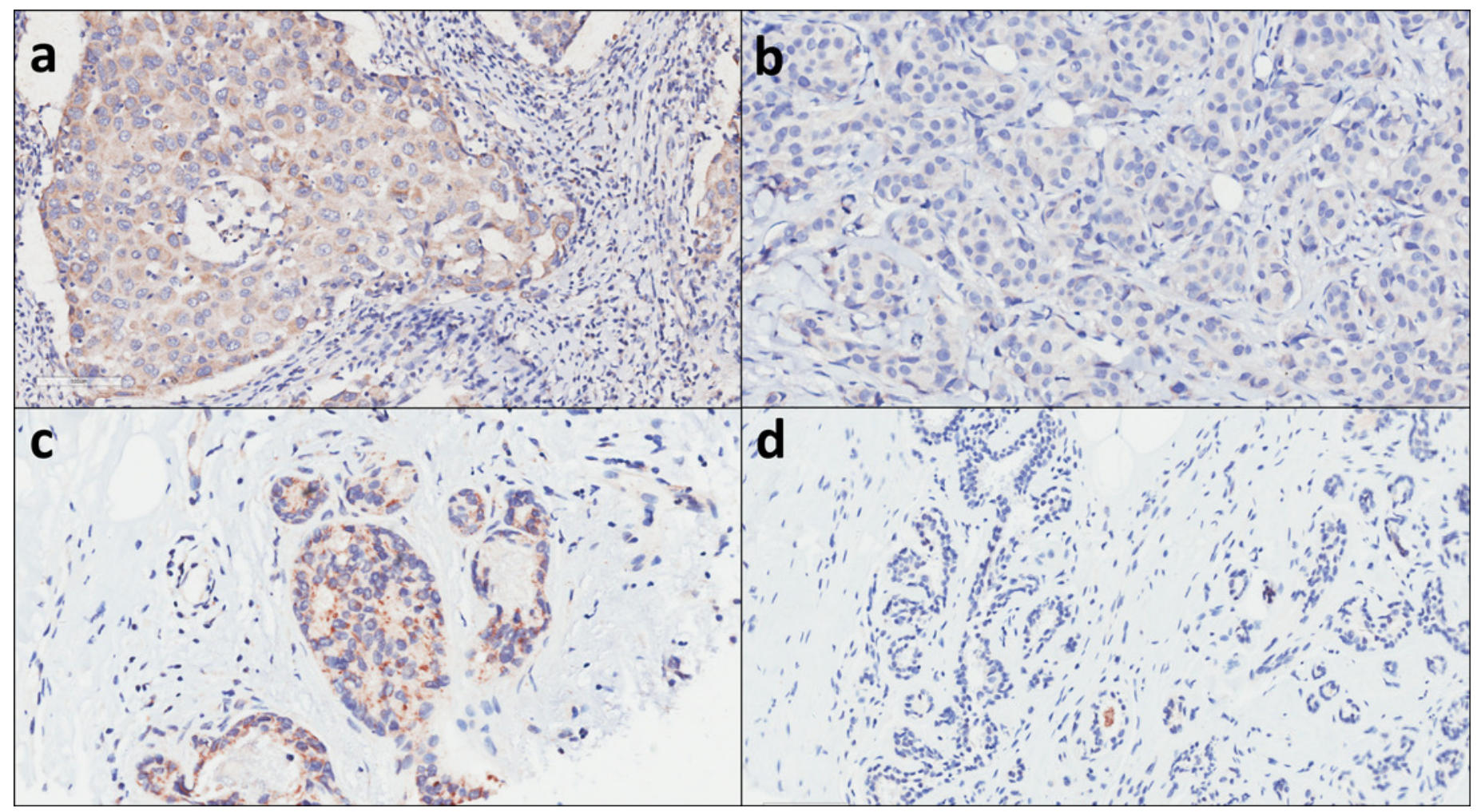


Figure 2

Kaplan-Meier curve of overall survival in relation to GADD45A protein expression.

(A) breast cancer; (B) TN subtype breast cancer.
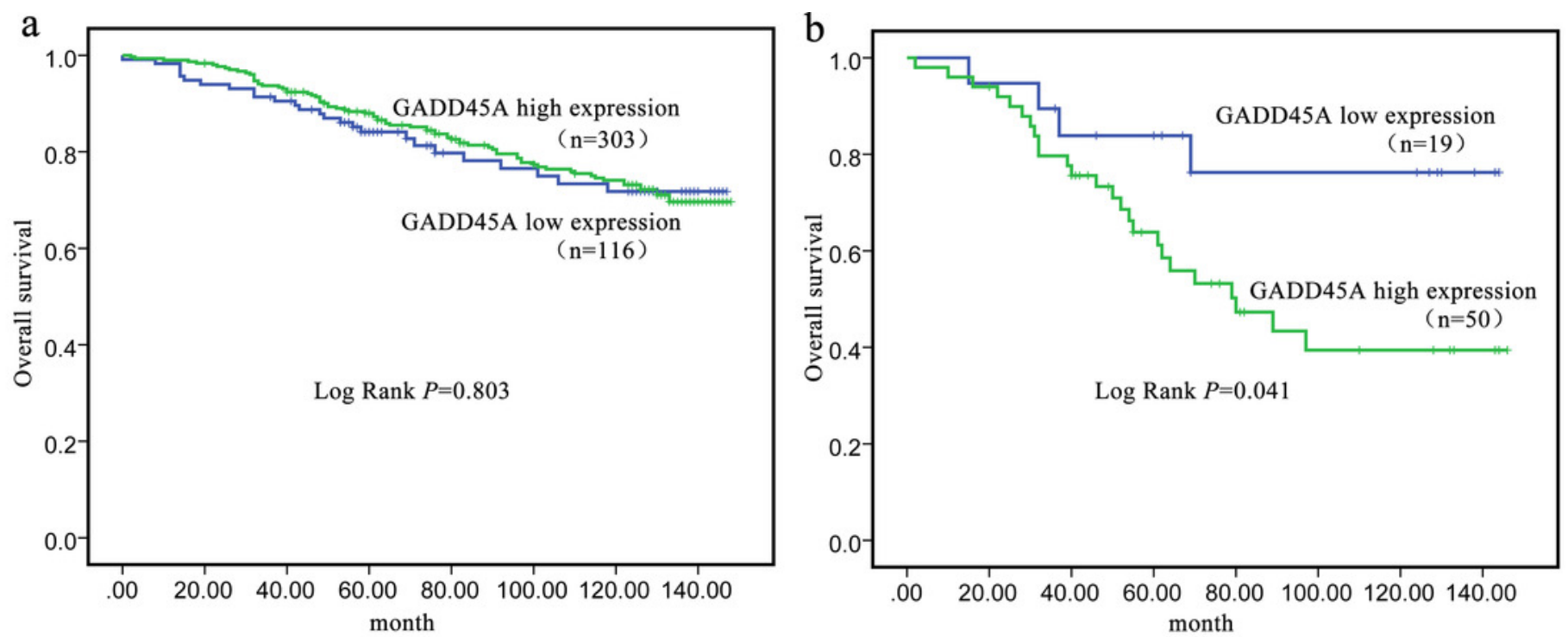